\title{
Ozono y COVID-19: bases fisiológicas y sus posibilidades terapéuticas según el estadio evolutivo de la infección por SARS-CoV-2
} Ozone and COVID-19: physiological bases and their
therapeutic possibilities according to the evolutionary
stage in SARS-CoV-2 infection

M. E. Fernández Cuadros¹, M. J. Albaladejo Florín , S. Álava Rabasa , D. Peña Lora² y O. S. Pérez Moro

${ }^{1}$ Medicina Física y Rehabilitación. Hospital Universitario Santa Cristina. Madrid, España. ${ }^{2}$ Unidad de Geriatría. Hospital Universitario Santa Cristina, Madrid, España

\section{RESUMEN}

No existe a la fecha un tratamiento definitivo para la nueva pandemia SARS-CoV-2. Están reconocidos tres estadios evolutivos en la infección por SARS-CoV-2 (infección temprana, fase pulmonar e hiperinflamación sistémica), con signos y síntomas clínicos característicos. Hay en marcha 80 ensayos experimentales internacionales que buscan un tratamiento efectivo para la pandemia COVID-19. De ellos, solo hay tres que consideran como alternativa de tratamiento la ozonoterapia (autohemoterapia mayor]. No existe ningún estudio que evalúe la insuflación rectal de ozono, a pesar de ser una técnica segura, barata, sin riesgos y que es una vía de administración sistémica (oxígeno-ozono $95 \%-5 \%$ y yue justifica la realización de ensayos clínicos para validar las propiedades teóricas del ozono en el manejo del SARS-CoV-2, dados los excelentes resultados observados en el manejo del ébola.

El ozono tiene demostradas cuatro propiedades biológicas que podrían ser de potencial utilidad teórica como terapia complementaria en las diferentes fases de la infección por SARS-CoV-2. El ozono podría inactivar el virus por oxidación indirecta (ROS y LOP) y podría estimular el sistema inmune celular y humoral, siendo útil en la fase de infección temprana (estadios 1 y 2a). El ozono puede mejorar el intercambio gaseoso, reducir la inflamación y modular el sistema antioxidante, por lo que sería útil en
Fernández Cuadros ME, Albaladejo Florín MJ, Álava Rabasa S, Peña Lora D, Pérez Moro OS. Ozono y COVID-19: bases fisiológicas y sus posibilidades terapéuticas según el estadio evolutivo de la infección por SARS-CoV-2. Rev Soc Esp Dolor. 2021;28(1):27-36

\section{ABSTRACT}

To date, there is no definitive treatment for the new SARS-CoV-2 pandemic. Three evolutionary stages are recognized in SARS-CoV-2 infection (early infection, pulmonary phase and systemic hyperinflammation), with characteristic clinical signs and symptoms. There are 80 international experimental trials underway seeking an effective treatment for the COVID-19 pandemic. Of them, there are only 3 that consider to Ozone Therapy as an alternative (major auto hemotherapy). There is no study that evaluates Rectal Ozone Insufflation, despite being a safe, cheap, risk-free technique and that it is a systemic administration route (Oxygen-Ozone $95 \%-5 \%$ ) and that justifies conducting clinical trials to validate the theoretical properties of Ozone in the management of SARS-CoV-2, given the excellent results observed in the management of ebola.

Ozone has 4 proven biological properties that could be of potential theoretical utility as a complementary therapy in the different phases of SARS-CoV-2 infection. Ozone could inactivate the virus by indirect oxidation (ROS and LOP) and could stimulate the cellular and humoral immune system, being useful in the early infection phase (stages 1 and 2a). Ozone can improve gas exchange, reduce inflammation, and modulate the antioxidant system, so it would be useful in the

Recibido: 17-02-2020

Aceptado: 17-02-2021

Correspondencia: Marcos Edgar Fernández Cuadros marcosefc@hotmail.com 
la fase de hiperinflamación o "tormenta de citocinas", y en la fase de hipoxemia y/o fallo multiorgánico (estadios $2 b$ y estadio 3 ).

Dada la actual pandemia, urge llevar a cabo un estudio experimental que confirme o descarte las propiedades biológicas del ozono y le permita así ser una terapia complementaria o compasiva para el manejo efectivo de la infección por SARS-CoV-2.

Palabras clave: Coronavirus, pandemia, ozono, SARSCoV-2, COVID-19. hyperinflammation or cytokine storm phase, and in the hypoxemia and / or multi-organ failure phase (stages $2 b$ and stage 3 ).

Given the current pandemic, it is urgent to carry out an experimental study to confirm or rule out the biological properties of Ozone and thus allow it to be a complementary or compassionate therapy for the effective management of SARS-CoV-2 infection.

Key words: Coronavirus, pandemic, ozone, SARSCoV-2, COVID-19.

\section{INTRODUCCIÓN}

Los coronavirus (CoV) son virus ARN grandes que deben su nombre a su forma esférica, de la que sobresalen unas espículas en forma de corona (proteína $\mathrm{S}$, básicamente conformada por cisteína y triptófano). Pueden causar patología en mamíferos, aves y humanos. Los coronavirus humanos se aislaron por primera vez en 1960. Se conocen siete tipos de coronavirus humanos; de ellos cuatro producen resfriado común (HCoV229E, HCoV-OC43, HCoV-NL63 y HCoV-HKU1). En este siglo XXI han aparecido otros tres nuevos coronavirus cuya patogenicidad es mucho más grave y que causan el síndrome respiratorio agudo severo (SARS): el SARS-CoV (China, 2002), el MERS-CoV (Oriente Medio, 2012) y el SARS-CoV-2 $(1,2]$.

En diciembre de 2019, en Wuhan, provincia de Hubei (China), se reportó un brote de casos de neumonía causado por un nuevo coronavirus, el SARS-CoV-2 o COVID-19 [3] . Desde China se diseminó en 3 meses a prácticamente todo el mundo, impactando sobre la economía y la salud de los países afectados. Esta pandemia ha generado un impacto sobre los sistemas sanitarios. A día de hoy (27 de diciembre del 2020) hay 81.047.499 casos confirmados en 213 países y más de 1.770.281 muertos (4).

La actual pandemia confirma que el SARS-CoV-2 es un virus de alta infectividad y con una alta capacidad de transmisión. Se transmite a través de las gotitas de Flügge de las secreciones respiratorias de una persona infectada [al exhalar, toser o estornudar], que son capaces de transmitirse a distancias de hasta 2 metros. Al caer estas gotitas de Flügge, se depositan en las superficies, donde pueden infectar a otras personas si tocan esos objetos o superficies con la mano y luego se la llevan a los ojos, la nariz o la boca (트).

A la alta tasa de infectividad se le suma el elevado tiempo de incubación (entre 1 y 14 días), lo que le proporciona una gran transmisibilidad presintomática. Además, tras la curación, la transmisión es también posible (15 dias postcuración). Existen casos de transmisión fecal-oral, que ocasiona síntomas digestivos (diarrea). No se han reportado casos de transmisión perinatal ni por la leche materna [ㅌ].
No existe hasta la fecha un tratamiento antiviral efectivo contra el SARS-CoV-2. Los fármacos antivirales y corticoides sistémicos utilizados en otras infecciones víricas no han demostrado validez para SARS-CoV-2 (ㄱ). Algunos autores destacan el uso de corticoides al inicio de la respuesta hiperinflamatoria y no en fases tempranas, donde su uso precoz podría favorecer la replicación viral []. Se ha utilizado exitosamente el remdesivir (antiviral ARN) para tratar el primer caso de SARS-CoV-2 en Estados Unidos (9). El remdesivir es el antiviral más prometedor actualmente investigado, aunque ni su eficacia ni su seguridad están establecidas (9). El lopinavir/ritonavir disminuyó significativamente la carga viral en un caso de SARS-CoV-2, aunque la eficacia de esta combinación no está establecida de forma definitiva (10). Varios estudios han encontrado que la hidroxicloroquina puede inhibir algunos pasos en la replicación de varios virus, incluido el coronavirus (11). La hidroxicloroquina tiene además efecto inmunomodulador, suprimiendo la producción y liberación de factor de necrosis tumoral $\alpha$ (TNF- $\alpha$ ) e interleucina (IL) 6 (12). Actualmente la eficacia, dosis y seguridad de la hidroxicloroquina para el tratamiento y prevención del SARS-CoV-2 no están establecidas, y se necesitan más datos sobre si su actividad in vitro se corresponde con la eficacia clínica (12). Una reciente publicación refiere que el uso combinado de hidroxicloroquina y azitromicina es más efectivo para la eliminación el virus (13).

Con el fin de suprimir distintas citocinas proinflamatorias se están iniciando diversos protocolos cuasi experimentales a nivel hospitalario para modular la respuesta inmunológica excesiva y así evitar el daño producido por la tormenta de citocinas, siendo en muchos casos este fenómeno el responsable en un subgrupo de pacientes del desenlace fatal en la infección por el SARS-CoV-2 (14).

Varios estudios (Cuba, Italia, Alemania, Rusia y España) y años de experiencia han demostrado que el ozono es capaz de modular la inflamación y el dolor, además de haber demostrado efecto bactericida, fungicida, virucida y antiparasitario $(15,16)$. Esas propiedades antimicrobianas han hecho que el ozono sea reconocido como un desinfectante tan efectivo que se usa en muchas plantas potabilizadoras de agua a nivel mundial (15). 
En este contexto, creemos razonadamente que el ozono tiene cabida en el manejo de la presente pandemia SARS-CoV-2, por lo que realizaremos una revisión sobre el tema y sus teóricas posibilidades terapéuticas.

Actualmente se están realizando cerca de 80 ensayos clínicos que buscan definir la mejor terapia para el manejo de la infección por SARS-CoV-2. De ellos, solo tres están dedicados al estudio del ozono sobre esta enfermedad y su potencial uso terapéutico (ㄴ).

El objetivo de esta revisión es analizar las bases fisiológicas y posibilidades terapéuticas del ozono sobre la infección por SARS-CoV-2 (COVID-19) según el estadio evolutivo, y quizás plantear su utilización como terapia complementaria en el tratamiento compasivo del COVID-19, usando como vía de administración sistémica la insuflación rectal, por ser la técnica más sencilla, segura y económica.

\section{FISIOPATOLOGÍA DEL SARS-CoV-2}

La respuesta inmune inducida por la infección de SARS-CoV-2 ocurre en dos fases (17): una primera en la que predomina la patogenicidad viral y una segunda en la que la patología principalmente se debe a la respuesta inmunitaria excesiva del huésped. El tránsito de una fase a otra es progresivo, tanto desde el punto de vista clínico como analítico (14).

Durante la incubación y en la fase no grave de la enfermedad, se requiere de una respuesta inmune adaptativa específica para intentar eliminar el virus y limitar la progresión a estadios más avanzados de la infección. La segunda fase comienza cuando la respuesta inmune protectora se altera. La disfunción del sistema inmunitario genera una disminución de linfocitos T CD3+ y CD4+ acompañada de un aumento de neutrófilos y un aumento del índice neutrófilos/linfocitos, especialmente en los casos más graves, por lo que es un signo predictivo de evolución tórpida (18).

La disfunción del sistema inmunitario origina una serie de respuestas inmunitarias desproporcionadas y negativas para los diferentes tejidos afectados. Se reclutan complejos de señalización que desarrollan una cascada molecular que activa el factor de transcripción nuclear $K \beta$ (NF-K $\beta$ ), el factor regulador de interferón 3 (IRF3) y la producción de interferones tipo I (IFN- $\alpha / \beta$ ), además de una serie de citocinas proinflamatorias (principalmente IL-1 $\beta, \mathrm{IL}-6$, factor estimulante de colonias de macrófagos [MCSF], IP-10, MCP-1, factor de crecimiento de hepatocitos [HGF], interferón $\gamma$ [IFN- $\gamma$ ] y TNF- $\alpha$ ]. El perfil de citocinas que se asocia con la gravedad del COVID-19 se asemeja al que se observa en la linfohistiocitosis hemofagocítica secundaria (LHHS) (19). La LHHS es un síndrome hiperinflamatorio poco conocido caracterizado por una hipercitocinemia fulminante e insuficiencia multiorgánica mortal y que se puede presentar en el 3,7-4,3\% de los casos de sepsis viral en adultos. La LHHS cursa con fiebre mantenida, citopenia, ferritina elevada y afectación pulmonar en un $50 \%$ de los pacientes (20). Según un estudio retrospectivo multicéntrico de 150 casos de COVID-19 en Wuhan, la mortalidad podría deberse a una hiperinflamación viral, y como marcadores de gravedad estarían el aumento de la ferritina y de la IL-6 (21). Otros marcadores predictores de enfermedad grave por SARS-CoV-2 son el dímero D elevado, la linfopenia y el aumento de troponina I ultrasensible [2己].

El tratamiento actual del COVID-19 es de mantenimiento, y la insuficiencia respiratoria por síndrome de dificultad respiratoria aguda (SDRA) es la principal causa de mortalidad. Un subgrupo de pacientes con COVID-19 grave podría desarrollar un síndrome de "tormenta de citocinas" (23). Es importante la identificación precoz y el tratamiento de la hiperinflamación utilizando todas las terapias existentes con perfiles de seguridad aceptables para abordar la necesidad inmediata de reducir la mortalidad (르).

\section{PRESENTACIÓN CLÍNICA DEL SARS-COV-2}

La infección por SARS-CoV-2 no progresa de forma similar en todas las personas que entran en contacto con él. Un pequeño porcentaje de personas expuestas no se infectan ni desarrollan síntomas clínicos (30\%). El interés en la clasificación clínica reside en poder proponer el abordaje terapéutico más adecuado para cada paciente según la fase en la que se encuentre (14).

Siddiqi y Mehra han propuesto clasificar las fases de la enfermedad en tres periodos (25):

- Estadio I (leve o infección temprana). Abarca desde la inoculación hasta el establecimiento temprano de la enfermedad. Se caracteriza por síntomas inespecíficos como malestar, fiebre, tos seca, cefalea, anosmia o ageusia. El hemograma puede revelar linfopenia y neutrofilia, sin otras anormalidades significativas (Tabla I) (25).

- Estadio II (moderado, con afectación respiratoria sin hipoxemia [lla] o con hipoxemia [Ilb]]. Establecimiento de enfermedad respiratoria con empeoramiento de síntomas y aparición de signos radiológicos de neumonía viral (infiltrados u opacidades "en vidrio deslustrado" característicamente bilaterales] y analíticos [agudización de la linfocitopenia, elevación moderada del dímero $D$ y de las transaminasas], así como el comienzo de elevación de marcadores sistémicos de inflamación pero no de forma exagerada (procalcitonina normal o baja). En esta etapa la mayoría de los pacientes con COVID-19 necesitarian ser hospitalizados para su observación y tratamiento (un $15 \%$ ). Un aspecto clínico relevante en este periodo es la aparición de hipoxemia como marcador del progreso de la infección y la necesidad de ventilación mecánica fruto de la hiperrespuesta inflamatoria pulmonar, que, de no ser interrumpida, llevará al siguiente estadio (Tabla I) (25).

- Estadio III (grave o de hiperinflamación sistémica). Clínicamente en esta fase se observa en muchos pacientes una afectación multiorgánica con una evolución progresiva hacia el empeoramiento e inestabilidad hemodinámica y sepsis. Este periodo se caracteriza por una elevación significativa de marcadores de inflamación sistémica (IL-2, IL-6, IL-7, factor estimulante de colonias de granulocitos, proteína inflamatoria de macrófagos $1-\alpha$, TNF- $\alpha$, proteína $C$ reactiva [PCR], ferritina y dímero-D]. La ferritina es el indicador clave de activación macrofágica. El dímero $D$ se eleva de manera similar a lo que ocurre en el sindrome anti- 
TABLA I

GRAVEDAD DE LA INFECCIÓN POR SARS-CoV-2 POR ESTADIOS, SIGNOS, SÍNTOMAS, TERAPIAS POTENCIALES Y PROPUESTA DE OZONOTERAPIA SEGÚN PROPIEDADES/EVOLUCIÓN DE LA ENFERMEDAD

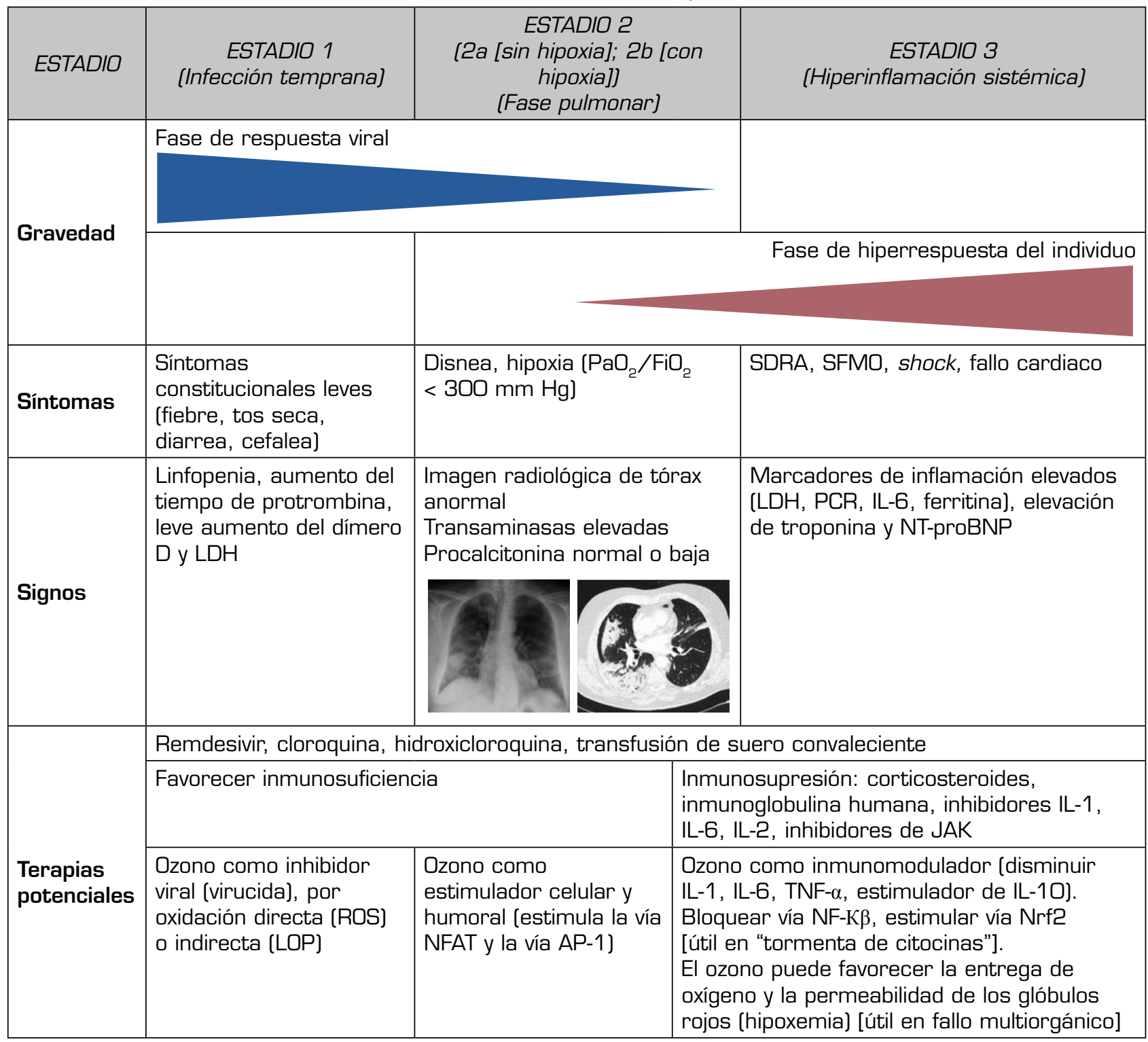

Modificada de Siddiqi y cols. (25)

AP-1: activated protein 1. FiO, fracción inspirada de oxígeno. IL: interleucina. JAK: cinasa de Janus. LDH: lactatodeshidrogenasa. LOP: productos de oxidación lipídica. NFAT: nuclear factor activated T-cells. NF-K $\beta$ : nuclear factor K $\beta$. Nrf2: nuclear eritroid factor 2. NT-proBNP: N-terminal pro-brain natriuretic peptide. $\mathrm{PaO}_{2}$ : presión arterial de oxígeno. PCR: proteína $C$ reactiva. ROS: especies reactivas de oxígeno. SDRA: síndrome de dificultad respiratoria aguda. SFMO: síndrome de fallo multiorgánico. TNF- $\alpha$ : factor de necrosis tumoral $\alpha$.

fosfolípido, junto con plaquetopenia y alteraciones de la coagulación (Tabla I] [25].

\section{OZONO Y PROPIEDADES MEDICINALES}

El ozono es el tercer agente oxidante más fuerte, después del flúor y del persulfato, un hecho que expli- ca su alta reactividad (26). Es la forma alotrópica o inestable de oxígeno [그). Se usa para tratar varias enfermedades infecciosas, autoinmunes, degenerativas y ortopédicas (28-30). Se postula que el ozono tiene propiedades analgésicas, antinflamatorias, inmunomoduladoras y tróficas (26]).

El ozono se descompone espontáneamente y, por lo tanto, apenas es almacenable. La concentración de 
ozono se reduce a la mitad a $30{ }^{\circ} \mathrm{C}$ en 25 minutos, a $20^{\circ} \mathrm{C}$ en 40 minutos y a $-50^{\circ} \mathrm{C}$ en 3 meses (26). El ozono se descompone rápidamente, dividiéndose en $\mathrm{O}_{2}$ y $0^{-}$( 0 monoatómico), que es muy reactivo y tiene propiedades antiinfecciosas, antivirales, antiparasitarias, antifúngicas y antitóxicas (31).

El ozono no es un medicamento homeopático; por el contrario, tiene una relación dosis/efecto $(\underline{26}, 32)$. La mayoría de los generadores médicos generan concentraciones de ozono desde 1 hasta 70-100 $\mu \mathrm{g} / \mathrm{ml}$. La dosis total de ozono es equivalente al volumen de gas (ml) multiplicado por la concentración de ozono $(\mu \mathrm{g} / \mathrm{ml})$. Se deben conocer las dosis óptimas del ozono para las diferentes aplicaciones médicas (26). Sin embargo, la ventana terapéutica está entre 10 y $80 \mu \mathrm{g} / \mathrm{ml}$ (31).

El ozono disuelto en el agua plasmática reacciona inmediatamente con una serie de biomoléculas y desaparece. Hay dos compuestos (especies reactivas de oxígeno o ROS y productos de oxidación lipídica o LOP] que representan los "mensajeros del ozono" y son los responsables de los efectos biológicos y terapéuticos. Los ROS se producen inmediatamente, en la fase inicial (principalmente peróxido de hidrógeno $\circ \mathrm{H}_{2} \mathrm{O}_{2}$ ), y son los responsables de los efectos biológicos tempranos sobre la sangre (eritrocitos, leucocitos, plaquetas). Por el contrario, los LOP, que se producen simultáneamente, tienen una vida media más larga, alcanzan el sistema vascular e interactúan con varios órganos, donde desencadenan efectos tardíos. Algunos de estos órganos diana son el hígado (en la hepatitis crónica) y el sistema vascular (en vasculopatías), mientras que otros órganos probablemente están involucrados en la restauración de la homeostasis normal (sistema nervioso central, tracto gastrointestinal, tejido linfoide asociado a la mucosa) $(26,30,32)$.

Los efectos biológicos del ozono provocados durante la exposición al plasma se deben a la formación de ROS y LOP (mensajeros de ozono). Los ROS mejoran la entrega de oxígeno en los eritrocitos. También estimulan los leucocitos y activan el sistema inmune. En las plaquetas el ozono favorece la liberación de citocinas (hormonas locales) y de factores de crecimiento. Los LOP aumentan la liberación de óxido nítrico en el endotelio. Los LOP favorecen la generación de eritrocitos superdotados y la liberación de células madre en la médula ósea. Finalmente, los LOP actúan sobre otros órganos, activando la regulación positiva de proteínas de choque oxidativo (OSP) y enzimas antioxidantes (superóxido dismutasa, catalasa y glutatión-peroxidasa) $(26,32)$.

El ozono es un agente capaz de modular eficazmente el estrés oxidativo, estabilizando el equilibrio prooxidante y antioxidante y también la inflamación (31). En resumen, el ozono podría aumentar las propiedades reológicas de la sangre y podría mejorar la entrega de oxígeno, la producción endotelial de óxido nítrico y la modulación de la inmunidad vía inducción de citocinas. En este sentido, el ozono es el inductor de citocinas "ideal" (Tabla II) $(\underline{26,32})$.

\section{PROPIEDADES POTENCIALES TEÓRICAS DEL OZONO SOBRE EL SARS-COV-2}

El ozono tiene muchas propiedades que podrían ser útiles frente a la infección por COVID-19 y sobre los diferentes estadios evolutivos de la infección. Babior y cols. han demostrado que nuestro sistema inmune es capaz de producir ozono para desarrollar la actividad bactericida (33). Bocci ha demostrado que el ozono es además activo frente a virus, hongos, levaduras y protozoarios $[26,32]$.

Se postulan cuatro propiedades teóricas por las que el ozono podría ser útil en el manejo del SARS-CoV-2, que se describen a continuación.

\section{Propiedad 1}

En una revisión de modelos experimentales, Rowen y cols. describen que el ozono es capaz de inactivar directamente muchos virus (virus de Norwalk, virus de hepatitis A, poliovirus y el colífago MS3) (34). La explicación del efecto "virucida" es que el ozono es capaz de oxidar la glucoproteína de su membrana, transformándola de la forma reducida (R-S-H) a la forma oxidada (R-S$\mathrm{S}-\mathrm{R}$ ). El virus normalmente requiere la forma reducida para entrar en las células e infectarlas (34). Mirazmi ha observado que el citomegalovirus pierde infectividad si su grupo tiol o sulfhidrilo (R-S-H) se oxida (R-S-S-R), como lo observó Rowen. Los coronavirus, así como el virus del Ébola, tienen en su proteína S de membrana (Spike) regiones ricas en cisteína y triptófano $(34,35)$. Rowen y

TABLA II

EFECTO DEL OZONO SOBRE LOS ÓRGANOS DIANA Y LAS MODIFICACIONES FUNCIONALES

\begin{tabular}{|c|l|l|l|}
\hline Sustrato & Mensajero & Órganos diana & \multicolumn{1}{c|}{ Modificaciones funcionales } \\
\hline \multirow{4}{*}{ Ozono en plasma } & \multirow{4}{*}{ ROS } & Eritrocitos & Mejora la entrega de oxígeno (vía 2,3-DPG) \\
\cline { 3 - 4 } & Leucocitos & Activación de la inmunidad \\
\cline { 2 - 4 } & Plaquetas & Liberación de citocinas y factores decrecimiento \\
\cline { 2 - 4 } & \multirow{4}{*}{ LOP } & Endotelio & Liberación de óxido nítrico y eritrocitos superdotados \\
\cline { 3 - 4 } & Médula ósea & Liberación de células madre \\
\cline { 3 - 4 } & Otros órganos & $\begin{array}{l}\text { Regulación de proteínas de shock oxidativo y enzimas } \\
\text { antioxidantes }\end{array}$ \\
\hline
\end{tabular}

Modificada de Bocci (26).

2,3-DPG: 2,3-difosfoglicerato. LOP: productos de oxidación lipídica. ROS: especies reactivas de oxígeno. 
Eren teóricamente postulan que el ozono oxidaría directamente los grupos tiol (R-S-H) de cisteína y triptófano a la forma R-S-S-H, inactivándolos y bloqueando su fusión directamente. Si el ozono no llega directamente, sus mensajeros (ozónidos) tipo ROS o LOP $\left(\mathrm{H}_{2} \mathrm{O}_{2}\right.$, superóxido, óxido nítrico, etc.) aún mantienen su poder oxidante para inactivar a los virus (Figura 1) (34-39). Si el ozono, por el proceso de peroxidación, daña la cápside, el ciclo reproductivo del virus se altera; ahí radicaría el teórico efecto terapéutico del ozono en la fase inicial (estadio 1) de la infección temprana (Tabla I y Figura 2).

\section{Propiedad 2}

El ozono, mediante segundos mensajeros $\left(\mathrm{H}_{2} \mathrm{O}_{2}\right)$, es capaz de estimular la inmunidad celular y humoral a través de la vía de señalización NFAT (nuclear factor activated T-cells) y de la vía AP-1 (activated protein-1) (40). Estas vías son factores de transcripción cruciales, ya que inducirían la expresión de genes para liberar citocinas inflamatorias (IL-2, IL-6, IL-8, TNF- $\alpha$ e IFN- $\gamma$ ) que producirán la inflamación que reclutará a los neutrófilos, linfocitos y macrófagos, a fin de realizar la fagocitosis para limitar la infección a ese nivel, matando a los patógenos locales $(\underline{26,40})$. Esta propiedad del ozono permitiría actuar en la fase de respuesta viral (estadio 1 y estadio 2a) de la infección viral (Tabla I y Figura 2).

\section{Propiedad 3}

El ozono, a dosis terapéuticas, modula el factor nuclear eritroide tipo 2 (Nrf2) y NF-K $\beta$ e induce el ree- quilibrio del ambiente antioxidante (40-45). El Nrf2 es el transcriptor clave que controla diversos aspectos relacionados con la homeostasis celular como respuesta a los agentes tóxicos u oxidantes. En particular, los efectos del Nrf2 median la transcripción basal o inducida de enzimas antioxidantes de fase ॥ (produce las enzimas catalasa, glutatión-peroxidasa y superóxido-dismutasa), que son las enzimas responsables de la eliminación de ROS. Durante los procesos inflamatorios agudos, para incrementar la magnitud de la respuesta, el NF-kB promueve el aumento de la actividad de la NADPH-oxidasa mitocondrial, la principal fuente endógena del radical anión superóxido. En la actualidad queda claro que existen fuertes nexos entre la actividad coordinada de activación de genes por parte de ambos factores de transcripción (NF-kB y Nrf2) para dar solución a procesos inflamatorios a nivel celular y de los tejidos. Un desequilibrio entre las rutas del NF-kB y Nrf2 se asocia con un gran número de enfermedades, como es el caso de las complicaciones del COVID-19 $(44,45)$. Fernández-Cuadros y cols. han descrito que el ozono podría bloquear la vía NF-K $\beta$, disminuyendo las citocinas proinflamatorias IL-1, IL-6 y TNF- $\alpha$, y estimular las citocinas antiinflamatorias IL-4 e IL-10 $(46,47)$. Además, en un reciente estudio, Fernández-Cuadros y cols. han observado que el ozono es capaz de modular la inflamación disminuyendo marcadores de inflamación como la PCR y la VSG (velocidad de sedimentación globular) (16). Por estas características, creemos que el ozono podría modular la inflamación y podría tener un papel terapéutico en la fase de hiperinflamación, actuando sobre la tormenta de citocinas (Tabla I y Figura 2).

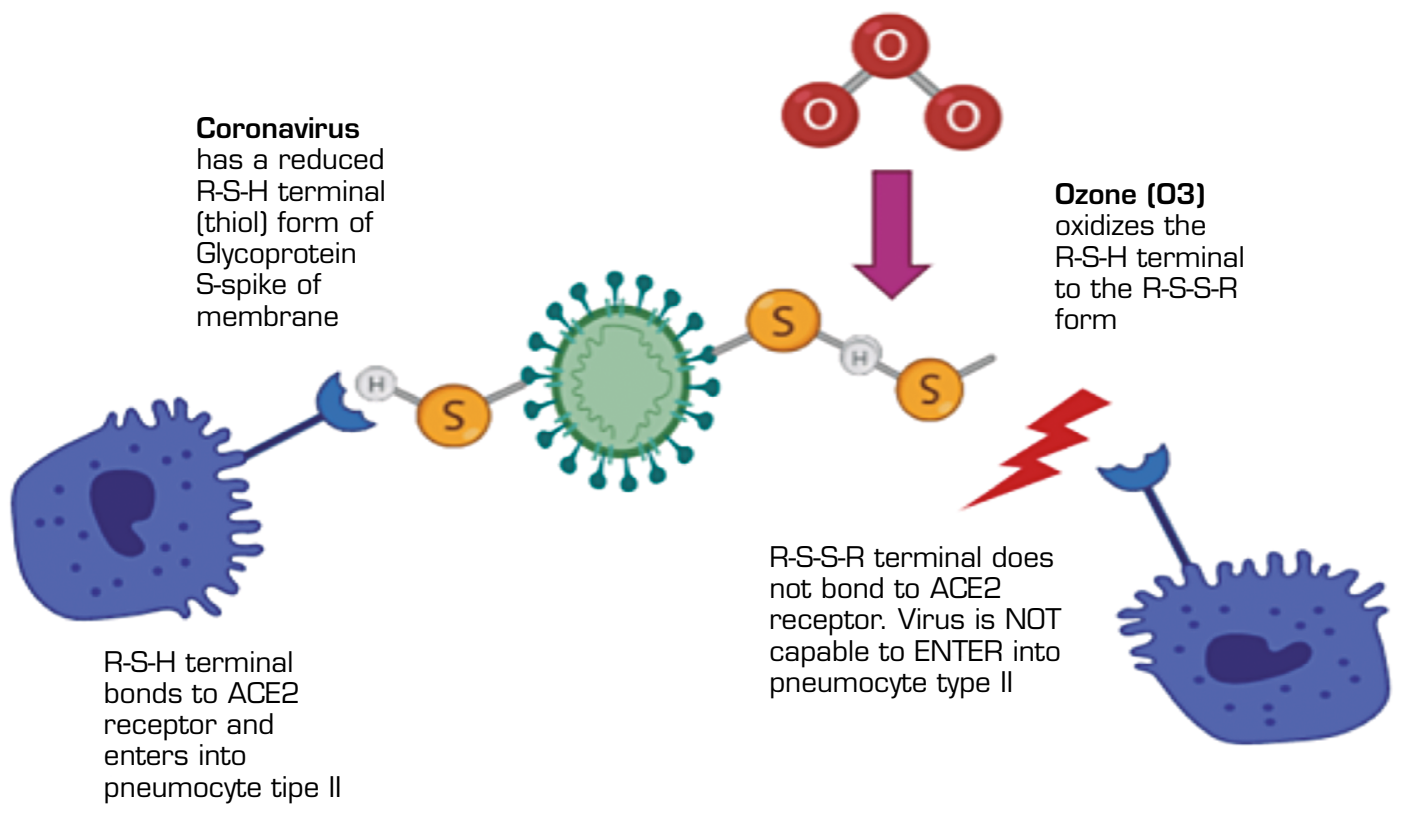

Fig. 1. El coronavirus debe su nombre a la proteína que lleva a su alrededor, que tiene forma de corona (proteína S, Spike] y que está constituida por cisteína y triptófano. El ozono o sus mediadores (ozónidos [ROS, LOP]) podrían ser potencialmente capaces de oxidar esos residuos, impidiendo su unión al receptor de ACE2 (angiotensin converting enzyme type 2) del neumocito tipo 2. Tomada de Fernández-Cuadros y cols. (399). 


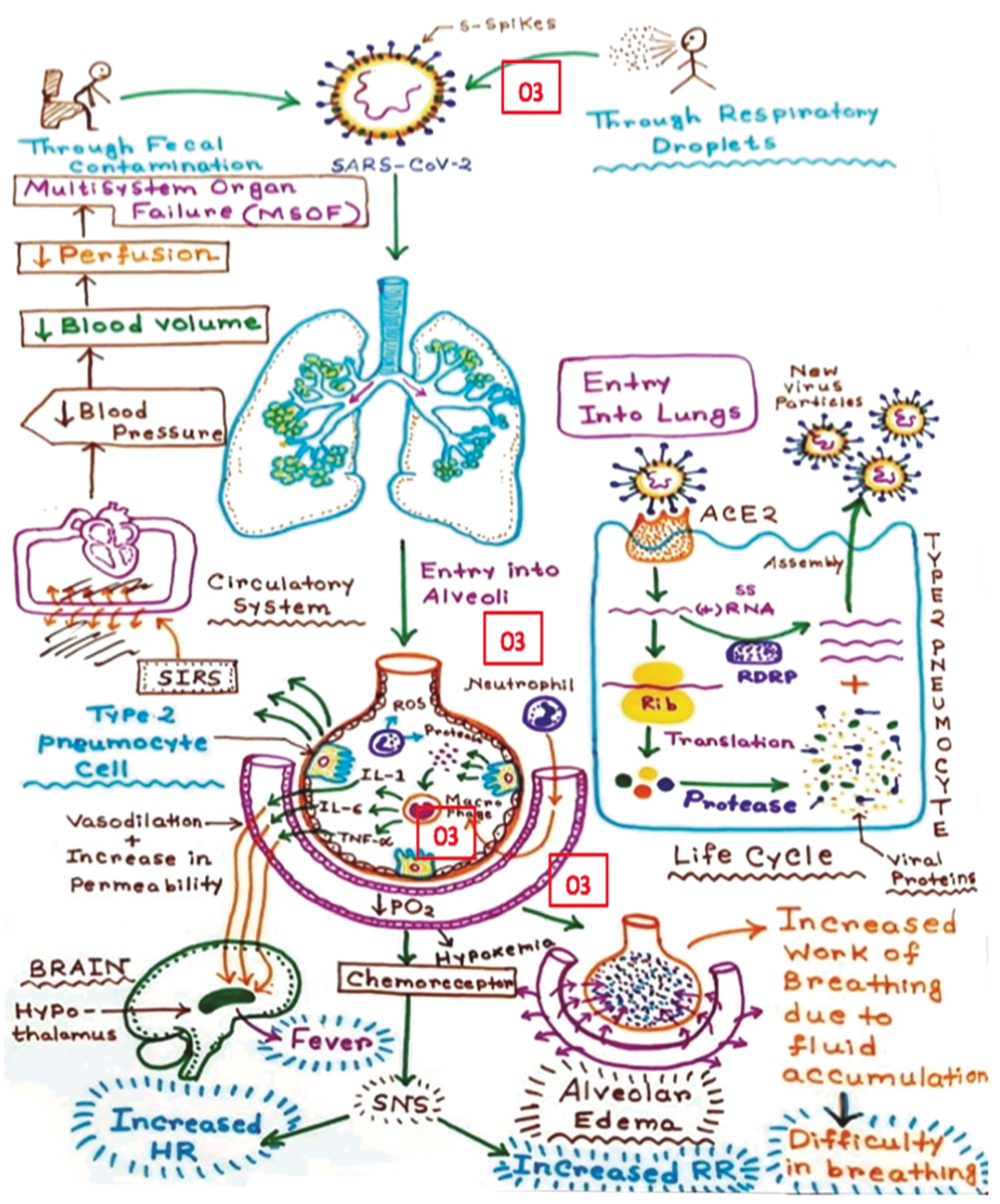

Fig. 2. Efectos potenciales del ozono $\left(\mathrm{O}_{3}\right.$ ): 1) Oxidación a nivel de la proteína $\mathrm{S}$ (Spike) rica en cisteína y triptófano. 2) Estimulación de la inmunidad celular y humoral (llamada a neutrófilos, macrófagos, etc. ). 3) Inhibición de IL-1, IL-6, TNF- $\alpha$ (vía NRf2, vía NF-Kß). 4) Mejoría de la hipoxemia (por disociación 2,3-DPG) y por mejoría de las propiedades reológicas de los glóbulos rojos (39).

\section{Propiedad 4}

El ozono podría mejorar la circulación y perfusión pulmonar y de todos los órganos en estado de hipoxia. Mejora el metabolismo del oxígeno $(15,26)$. Los eritrocitos ozonizados muestran una glucólisis mejorada con un aumento de los niveles de trifosfato de adenosina (ATP) y 2,3-difosfoglicerato [2,3-DPG], que pueden desplazar la curva de disociación de $\mathrm{HbO}_{2}$ hacia la derecha, aumentar la $\mathrm{PO}_{2}$ arterial y disminuir la $\mathrm{PO}_{2}$ venosa (efecto Bhor), mejorando el suministro de oxígeno a los tejidos isquémicos $(15,26)$. Aplicaciones continuas de ozono estimulan la médula ósea y la inducen a generar nuevos eritrocitos "superdotados" con incremento en el contenido de 2,3-DPG, así como una elevación de la glucosa-6-fosfato-deshidrogenasa, lo que puede posibilitar una profunda modificación de las actividades funcionales conduciendo a los tejidos y órganos de un estado hipóxico a uno normóxico $(15,26)$. Los pacien- tes con SARS-CoV-2 son propensos a tener hepatitis leve no específica, fibrosis pulmonar e insuficiencia renal (48). La terapia con ozono estabiliza el metabolismo hepático, y los niveles plasmáticos de fibrinógeno y protrombina tienden a normalizarse en pacientes infectados, lo que sugiere una mejora en la síntesis de proteínas hepáticas (48). Hay bastantes investigaciones que demuestran el efecto protector del ozono para prevenir el daño oxidativo al corazón, hígado, pulmón y tejido renal (48). Por estos motivos, creemos que el ozono tendría utilidad en la fase de hiperinflamación y fallo multiorgánico, restaurando la hipoxemia [Tabla I y Figura 2).

\section{MÉTODO DE ADMINISTRACIÓN}

El ozono se puede administrar por diferentes vías, ya sean sistémicas (autohemoterapia mayor, autohe- 
moterapia menor, solución salina saturada, insuflación rectal y vaginal) o no sistémicas (ozonoterapia por bolsa) $(26,31)$. Existen de momento tres ensayos clínicos que se están llevando a cabo en China y buscan valorar la efectividad de la autohemoterapia mayor sobre el SARS-CoV-2 (4). No existe ningún estudio experimental que esté valorando la administración de ozono sistémico por técnica de insuflación rectal. En esta revisión proponemos que una vía sistémica de fácil realización para la administración de ozono es la rectal.

El método de insuflación rectal es una técnica de aplicación sistémica del ozono que entrega un 95-5\% de oxígeno-ozono, pero sin los riesgos ni las dificultades técnicas que implica la extracción venosa, la ozonización y la reinfusión venosa. La técnica propuesta, de acuerdo con la Declaración Internacional de Ozonoterapia de Madrid, es administrar un volumen de 100 $300 \mathrm{ml}$ de ozono rectal a una concentración de $10 \mu \mathrm{g} /$ $\mathrm{ml}$, para ir subiendo progresivamente cada 3 días hasta alcanzar una concentración máxima de 35-39 $\mu \mathrm{g} / \mathrm{ml}$ en un tiempo previsto de diez sesiones totales (empezar a bajas concentraciones y subir lentamente) (49). Basados en estas recomendaciones, proponemos un volumen de $100 \mathrm{ml}$ y una concentración de $35 \mu \mathrm{g} / \mathrm{ml}$ a fin de no superar los valores que establece la Declaración de Madrid (49).

El Dr. Rowen, con la misma técnica de administración (ozono rectal), aplicada a cinco pacientes con ébola (enfermedad que presenta una mortalidad del $60 \%$ ), ha reportado una tasa de éxito del $100 \%$. Rowen observó mejoría desde la primera insuflación, aunque completó hasta cinco sesiones de tratamiento. Este alentador reporte justifica la realización de ensayos clínicos para valorar las propiedades teóricas del ozono en el manejo del SARS-CoV-2 (50).

\section{CONCLUSIONES}

No existe a la fecha un tratamiento definitivo para la nueva pandemia SARS-CoV-2. Hay en marcha 80 ensayos experimentales internacionales que buscan un tratamiento efectivo para la pandemia COVID-19. De ellos, solo hay tres que consideran como alternativa de tratamiento la ozonoterapia [autohemoterapia mayor). No existe ningún estudio que evalúe la insuflación rectal de ozono, a pesar de ser una técnica segura, barata, sin riesgos y de ser una vía de administración sistémica (ozono 95-96\%), lo que justifica la realización de ensayos clínicos para valorar las propiedades teóricas del ozono en el manejo del SARS-CoV-2, dados los excelentes resultados observados en el manejo del ébola.

El ozono tiene demostradas cuatro propiedades biológicas que podrían permitir su uso como terapia complementaria en las diferentes fases de la infección por SARS-CoV-2. El ozono podría inactivar el virus teóricamente por oxidación indirecta (ROS y LOP) y podría estimular el sistema inmune celular y humoral, siendo útil en la fase de infección temprana (estadios 1 y 2a). El ozono mejora el intercambio gaseoso, reduce la inflamación y modula el sistema antioxidante, por lo que sería útil en la fase de hiperinflamación o "tormenta de citocinas", y en la fase de hipoxemia y/o fallo multiorgánico (estadios $2 b$ y estadio 3).
Dada la actual pandemia, urge llevar a cabo un estudio experimental que confirme o descarte las propiedades biológicas del ozono y permita así que sea una terapia complementaria o compasiva para el manejo efectivo de la infección por SARS-CoV-2.

\section{BIBLIOGRAFÍA}

1. Yin $Y$, Wunderink RG. MERS, SARS and other coronaviruses as causes of pneumonia. Respirology. 2018 Feb;23(2): 1307. DOI: 10.1111/resp.13196.

2. Gorbalenya AE, Baker SC, Baric RS, de Groot RJ, Drosten C, Gulyaeva AA, et al. Severe acute respiratory syndromerelated coronavirus: the species and its viruses: a statement of the Coronavirus Study Group. bioRxiv. 2020; published online Feb 11. DOI: 10(2020.02), 07-937862.

3. Chen N, Zhou M, Dong X, Qu J, Gong F, Han Y, et al. (2020). Epidemiological and clinical characteristics of 99 cases of 2019 novel coronavirus pneumonia in Wuhan, China: a descriptive study. Lancet. 2020;395(10223):507-13.

4. WHO. Coronavirus disease (COVID-2019) situation reports. 2020. [Consultado 27 diciembre 2020].

5. Hung LS. The SARS epidemic in Hong Kong: what lessons have we learned? J R Soc Med. 2003;96(8):374-8. DOI: 10.1258/jrsm.96.8.374.

6. Chen N, Zhou M, Xuan Dong X, Jieming Qu J, Fengyun Gong $F$, Yang Han et at. Epidemiological and clinical characteristics of 99 cases of 2019 novel coronavirus pneumonia in Wuhan, China: a descriptive study. Lancet. 2020;395:507-13. DOI: 10.1016/ S0140- 6736(20)30211-7.

7. Li H, Zhou Y, Zhang M, Wang H, Zhao Q, Liu J. Updated approaches against SARS-CoV-2. Antimicrob Agents Chemother. 2020;64(6):e00483-20. DOI: 10.1128/ AAC.00483-20.

8. Russell CD, Millar JE, Baillie JK. Clinical evidence does not support corticosteroid treatment for 2019-nCoV lung injury. Lancet. 2020;395(10223):473-5. DOI: 10.1016/S01406736(20)30317-2.

9. Wang M, Cao R, Zhang L, Yang X, Liu J, Xu, M, et al. Remdesivir and chloroquine effectively inhibit the recently emerged novel coronavirus (2019-nCoV) in vitro. Cell Res. 2020;30(3):269-71.

10. Lim J, Jeon S, Shin HY, Kim MJ, Seong YM, Lee WJ, et al. Case of the Index Patient Who Caused Tertiary Transmission of COVID-19 Infection in Korea: the Application of Lopinavir/Ritonavir for the Treatment of COVID-19 Infected Pneumonia Monitored by Quantitative RT-PCR. J Korean Med Sci. 2020;35(6):e79. DOI: 10.3346/ jkms.2020.35.e79.

11. Vincent MJ, Bergeron E, Benjannet S, Erickson BR, Rollin PE, Ksiazek TG, et al. Chloroquine is a potent inhibitor of SARS coronavirus infection and spread. Virol J. 2005;2:69. DOI: 10.1186/1743-422X-2-69.

12. Devaux CA, Rolain JM, Colson P, Raoult D. New insights on the antiviral effects of chloroquine against coronavirus: what to expect for COVID-19? Int J Antimicrob Agents. 2020;55(5):105938. DOI: 10.1016/j.ijantimicag.2020.105938.

13. Gautret P, Lagier JC, Parola P, Hoang VT, Meddeb L, Mailhe $M$, et al. Hydroxychloroquine and azithromycin as a treatment of COVID-19: results of an open-label non-randomized clinical trial. Int J Antimicrob Agents. 2020;56(1):105949. DOI: 10.1016/j.ijantimicag.2020.105949. 
14. Carrillo B, Martínez E. COVID-19 y acupuntura: ¿existe una nueva línea de investigación terapéutica? Rev Dig Acup. 2020;3(1):1-21.

15. Fernández-Cuadros ME, Pérez-Moro OS, Albaladejo-Florín MJ. Ozone fundamentals and effectiveness in knee pain: Chondromalacia and knee osteoarthritis. Germany: Lambert Academic Publishing; 2016.

16. Fernández-Cuadros ME, Pérez-Moro OS, Albaladejo-Florín MJ, Álava-Rabasa S. El ozono intraarticular modula la inflamación, mejora el dolor, la rigidez, la función y tiene un efecto anabólico sobre la artrosis de rodilla: estudio cuasiexperimental prospectivo tipo antes-después, 115 pacientes. Rev Soc Esp Dolor. 2020;27(2):78-88. D0I: 10.20986/ resed.2020.3775/2019.

17. Shi Y, Wang Y, Shao C, Huang J, Gan J, Huang X, et al. COVID-19 infection: the perspectives on immune responses. Cell Death Differ. 2020;27(5):1451-4. DOI: 10.1038/ s41418-020-0530-3.

18. Qin C, Zhou L, Hu Z, Zhang S, Yang S, Tao Y, et al. Dysregulation of immune response in patients with COVID-19 in Wuhan, China. Clin Infect Dis. 2020;71(15):762-8. DOI: 10.1093/cid/ciaa248.

19. Huang C, Wang Y, Li X, Ren L, Zhao J, Hu Y, et al. Clinical features of patients infected with 2019 novel coronavirus in Wuhan, China. Lancet. 2020;395(10223):497-506. DOI: 10.1016/S0140-6736(20)30183-5.

20. Karakike E, Giamarellos-Bourboulis EJ. Macrophage activation-like syndrome: a distinct entity leading to early death in sepsis. Front Immunol. 2019;10:55. DOI: 10.3389/ fimmu.2019.00055.

21. Ruan $Q$, Yang K, Wang W, Jiang L, Song J. Clinical predictors of mortality due to COVID-19 based on an analysis of data of 150 patients from Wuhan, China. Intensive Care Med. 2020;46(5):846-8. DOI: 10.1007/s00134-02005991-x.

22. Zhou F, Yu T, Du R, Fan G, Liu Y, Liu Z, et al. Clinical course and risk factors for mortality of adult inpatients with COVID-19 in Wuhan, China: a retrospective cohort study. Lancet. 2020;395(10229):1054-62.

23. Shimabukuro-Vornhagen A, Gödel P, Subklewe M, Stemmler HJ, Schlößer HA, Schlaak M, et al. Cytokine release syndrome. J Immunother Cancer. 2018;6(1):56. DOI: 10.1186/ s40425-018-0343-9.

24. Mehta P, McAuley DF, Brown M, Sánchez E, Tattersall RS, Manson JJ; HLH Across Speciality Collaboration. UK. COVID-19: consider cytokine storm syndromes and immunosuppression. Lancet. 2020;395(10229):1033-4. DOI: 10.1016/S0140-6736(20)30628-0.

25. Siddiqi HK, Mehra MR. COVID-19 illness in native and immunosuppressed states: a clinical- therapeutic staging proposal. J Heart Lung Transplant. 2020;39(5):405-7. DOI: 10.1016/j.healun.2020.03.012.

26. Bocci V. Ozone: a new medical drug. $2^{\mathrm{a}}$ ed. Dordrecht, the Nederlands, Germany: Springer Verlag; 2011.

27. lliakis E, Valadakis V, Vynios D H, Tsiganos C. P, Agapitos E. Rationalization of the activity of medical ozone on intervertebral disc. A histological and biochemical study. Riv Neuroradiol. 2001;14(1 suppl):23-30.

28. Paoloni M, Di Sante L, Cacchio A, Apuzzo D, Marotta S, et al. Intramuscular oxygen-ozone therapy in the treatment of acute back pain with lumbar disc herniation: a multicenter, randomized, double-blind, clinical trial of active and simulated lumbar paravertebral injection. Spine. 2009;4(13):1337-44.
29. Seyman D, Ozen NS, Inan D, Ongut G, Ogunc D. Pseudomonas aeruginosa septic arthritis of knee after intra-articular ozone injection. New Microbiol. 2012;35(3):345-8.

30. Mawsouf N, El-Sawalhi MM, Shaheen AA, Darwish HA, Martínez-Sánchez, et al. Effect of ozone therapy on redox status in experimentally induced arthritis. Rev Esp Ozono. 2011;1(1):32-43.

31. Madrigal C. Tratado de ozonoterapia. Madrid: Editorial La Salud Naturalmente; 2007.

32. Bocci VA. Scientific and medical aspects of ozone therapy. State of the art. Arch Med Res. 2006 May;37(4):425-35. DOI: 10.1016/j.arcmed.2005.08.006.

33. Babior BM, Takeuchi C, Ruedi J, Gutiérrez A, Wentworth P. Investigating antibody-catalyzed ozone generation by human neutrophils. Proc Natl Acad Sci U S A. 2003 Mar 18;100(6):3031-4. DOI: 10.1073/pnas.0530251100.

34. Rowen RJ, Robins H. A plausible "penny" costing effective treatment for corona virus ozone therapy. $J$ Infect Dis Epidemiol. 2020;6:113. DOl: 10.23937/2474-3658/1510113.

35. Eren E, Saribek F, Dalayci MZ, Yilmaz N. How to cripple SARSCOV-2 virus with Ozone treatment Thiol groups in viruses and SARS-COV-2. Disponible en: https://www.researchgate. net/publication/340253197_How_to_cripple_SARS-COV-2_ virus_with_Ozone_treatment_Thiol_groups_in_viruses_and_ SARS-COV-2 [último acceso 23 Feb 2021] DOI: 10.13140/ RG.2.2.30726.40004.

36. Schoeman D, Fielding BC. Coronavirus envelope protein: Current knowledge. Virol J. 2019;16:69.

37. Madu IG, Belouzard S, Whittaker GR. SARS-coronavirus spike S2 domain flanked by cysteine residues C822 and C833 is important for activation of membrane fusion. Virology. 2009;393:265-71.

38. Sharma VK, Graham NJ. Oxidation of amino acids, peptides and proteins by ozone: a review. Ozone Sci Eng. 2010;32(2):81-90.

39. Fernández-Cuadros ME, Albaladejo-Florín MJ, Peña-Lora D, Álava-Rabasa S, Pérez-Moro OS. (2020). Ozone (03) and SARS-CoV-2: Physiological bases and their therapeutic possibilities according to COVID-19 Evolutionary Stage. SN Compr Clin Med. 2020;1-9. DOI: 10.1007/s42399-020-00328-7.

40. Reth M. Hydrogen peroxide as second messenger in lymphocyte activation. Nat Immunol. 2002;3:1129-34.

41. Martínez-Sánchez G. Mechanisms of action of 03. Genomic pathways. Ozone Therapy Global Journal. 2019;9[1]:21-22.

42. Delgado-Roche L, Riera-Romo M, Mesta F, Hernández-Matos Y, Barrios JM, Martínez-Sánchez G. Medical Ozone Promotes Nrf2 Phosphorylation Reducing Oxidative Stress And Proinflammatory Cytokines In Multiple Sclerosis Patients. Rev Esp Ozono. 2018;8(2 Supp 1):48-9.

43. Martínez-Sánchez G, Delgado-Roche L. Up-date on the mechanisms of action of ozone through the modification of cellular signaling pathways. Role of Nrf2 and NFkb. Rev Esp Ozono. 2017;7(2):17-8.

44. Bocci V, Valacchi G. Nrf2 activation as target to implement therapeutic treatments. Front Chem. 2015;3:4.

45. Re L, Martínez-Sánchez G, Bordicchia M, Giuseppe Malcangi G, Pocognoli A, Morales-Segura MÁ, et al. Is ozone preconditioning effect linked to Nrf2/EpRE activation pathway in vivo? A preliminary result. Eur J Pharmacol. 2014;742:15862. DOI: 10.1016/j.ejphar.2014.08.029.

46. Fernández-Cuadros ME, Pérez-Moro OS, Mirón-Canelo JA. Could ozone be used as a feasible future treatment in osteoarthritis of the knee. Divers Equal Health Care. 2016;13(3):232-9.

47. Fernández-Cuadros ME, Pérez-Moro OS, Albaladejo-Florín MJ, Álava-Rabasa S, Tobar-Izquierdo M, Rodríguez-de-Cía J. 
A new paradigm for the management of knee osteoarthritis: the role of hyaluronic acid, platelet-rich plasma (PRP) and ozone in the modulation of inflammation. Science Repository. 2020; In press. DOI: 10.31487/j.JSR.2020.01.01.

48. Martínez-Sánchez G, Schwartz, A, Donna VD. Potential cytoprotective activity of ozone therapy in SARS-CoV-2/ COVID-19. Antioxidants. 2020;9(5):389.
49. Schwartz A, Sánchez GM, Sabah F. Declaración de Madrid sobre la ozonoterapia. $2^{a}$ ed.; Madrid; 2015.

50. Rowen RJ. Ozone and oxidation therapies as a solution to the emerging crisis in infectious disease management: a review of current knowledge and experience. Med Gas Res. Oct-Dec 2019;9(4):232-7. DOI: 10.4103/2045-9912. 273962. 